\title{
Multiple error correction towards optimisation of energy in sensor network
}

\author{
Samirah Razali, Kamaruddin Mamat, Nor Shahniza Kamal Bashah \\ Faculty of Computer and Mathematical Sciences, Universiti Technology of MARA, Shah Alam, Malaysia
}

\begin{tabular}{l}
\hline \hline Article Info \\
\hline Article history: \\
Received Sep 15, 2018 \\
Revised Dec 10, 2018 \\
Accepted Dec 25, 2018 \\
\hline
\end{tabular}

\section{Keywords:}

HARQ

Kalman filter

Remaining energy

SNR channel adaptation

Wireless sensor network

\begin{abstract}
Hybrid ARQ (HARQ) is among the optimum error controls implemented in Wireless Sensor Network as it reduces the overhead from retransmission and error correcting codes. The advancement in WSN includes the usage of high number of nodes and the increase in traffic with large data transmitted among the nodes had concerned the need for a new approach in error control algorithm. This paper proposed the multiple error correction based on HARQ process to aid the changes in channel with proper error correction assignment towards optimising the performances of WSN in terms of bit error rates, remaining energy, and latency for different types of congestion and channel conditions. In this study, we have developed the channel adaptation algorithm that can adapt to sudden changes and demonstrated the optimal error correcting codes as well as adjustment on the transmit power for the given channel condition and congestion presented. From the result analysed, the optimisation between the remaining energy and Bit Error rates happened on the basis of adapting to these different channel condition and congestion to minimize redundancies appended. From the result obtained, we concluded that by using multiple error correction algorithm with the aid of adjustment on the transmit power, the remaining energy can be optimised together with Bit Error rates and the excessive redundancies can be reduced.
\end{abstract}

\section{Corresponding Author:}

Kamaruddin Mamat,

Faculty of Computer and Mathematical Sciences,

Universiti Technology of MARA,

Shah Alam, Malaysia.

Email: kamar@tmsk.uitm.edu.my

\section{INTRODUCTION}

The Wireless Sensor Network (WSN) is very crucial in monitoring field such as habitat monitoring, environmental, agricultural, military, and tracking field. Recently, there are some emerging applications of WSN in big data [1],[2] and internet of things (IoT) [3],[4]. The existing technology of WSN critically benefits in terms of cost, scalability, and also provide supports towards human-work constrained when monitoring dangerous places such as natural disasters and unfriendly environments [5].

As WSN is energy-constrained and error-prone, researchers have established many methods and ways to overcome these problems. Researches back then provided the method to reduce energy consumption through calibrating or adjusting the transmission power such as by estimating the Signal to Noise Ratio (SNR) [6] and Received Signal Strength Indicator RSSI [7] using Kalman Filter (KF) in order to adjust the transmission power. The important aspect of high error rates in a network cannot be cast aside although minimizing the energy usage using transmission power control (TPC) is an effective method to maintain the lifetime as high error rates cause retransmission to flood the network and this consumes more energy. Thus, the problem of high energy usage needs to be tackled alongside with high error rates. In addition, some 
techniques in implementing error correction schemes such as HARQ indirectly reduce error rates and possibly reduce the energy consumption by reducing the flooding of retransmission in a congested network. There are related studies on the effect of HARQ with BCH in Coded Division Multiple Acess (CDMA) WSN towards the error rates and energy consumption [8],[9].

The authors reported on high Bit Error Rates (BER) at low hop count. Thus, the architecture or node deployment contributes to the increase in error rates or packet corruption in the presence of interferences and noises particularly when the nodes are located within the transmission range of other nodes. Practically, the nodes are usually deployed in a non-uniform manner following the geographical structure. Thus, there can be variations of node densities in one monitoring area. Moreover, apart from interferences and signal fading, the channel condition might change over time due to reflections and refractions of signals and also might be contributed from the hardware itself. Thus, the implementation of existing error correction schemes might not be reliable as different levels of congestions might present in one monitoring area with sudden changes of interferences and noise over time. The work of [10] introduced the multi-coding schemes for WSN where different coding schemes were implemented at the nodes and the sink. Based from this literature, it was demonstrated that the multi-coding schemes increased the lifetime of a network. However, further studies on the optimal codeword length and error correcting capability that corresponds to the presence of noises are needed in order to fully optimize the energy usage and error rates in a network.

Different coding schemes and error correcting capability will append different number of redundancies to the transmitted bits. Based on our previous studies [11]-[13], we demonstrated that high redundancy consumed too much energy in decoding the transmitted data. Meanwhile, a network with bad condition and high error rates might not able to handle large number of erroneous bits due to the relatively low error correcting capability. Thus, in this paper, we proposed the approach to optimize the energy usage with BER by modifying the Hybrid ARQ (HARQ) process aided with multi-coding schemes and power control that can adapt to the changes in channel condition that were estimated as SNR using KF. Our proposed algorithm merits on its capability to adapt to the changes in SNR in which every SNR range describe different error correction used and transmit power calibration to minimise the higher redundancies when these excessive redundancies are not needed in better SNR condition and vice versa. Transmit power calibration aided to reduce the interferences and noises in noisy channel when SNR is low as the ratio of noises will rise the BER sharply. We presented the comparisons between our proposed algorithm towards the increase in performances with the existing error correction used in Section 3 in which we able to reduce high BER in low SNR condition and maintain remaining energy in high SNR condition.

\section{RESEARCH METHOD}

The development of Multiple Error Correction (MEC) consists of several methods. First, we proposed the MEC algorithms based on the Hybrid ARQ (HARQ) process. Second, we aided the algorithm by calibrating the transmit power according to the MEC assigned for different error correcting codes at different SNR range. Lastly, we integrated Kalman Filter (KF) to estimate the SNR values for incoming transmission in order to avoid wrong assignment of error correcting codes towards the sudden changing environment of CDMA WSN.

Figure 1 shows the chronological development of our proposed algorithm. We also carried out three different preliminary experiments comprising the simulation of CDMA WSN with no error correction, with a range of low error correcting capability, and with a range of high error correcting capability. We selected $\mathrm{BCH}$ and RS codes as the error correcting codes in our algorithm based on the preliminary experiments where $\mathrm{BCH}$ and RS codes optimized the performance of CDMA WSN compared to the Convolutional Codes [11],[12]. Some studies also agreed that the RS [14] and BCH codes [15] are among other optimum error correcting codes which provide reliability during transmission. From our preliminary testing [11]-[13], we observed that in a low SNR condition with high BER, the use of low error correcting capability might not be able to solve the problem of high BER. Meanwhile, the use of high error correcting capability caused the problems of increment in energy consumption as well as latency. We also found that medium error correcting capability for the high SNR condition might be able to optimize BER performance as well as the remaining energy. Besides that, the use of codeword length corresponding to the number of generated bits in the network is also critical. Higher numbers of codeword lengths (more than 127) promote more energy usage. 


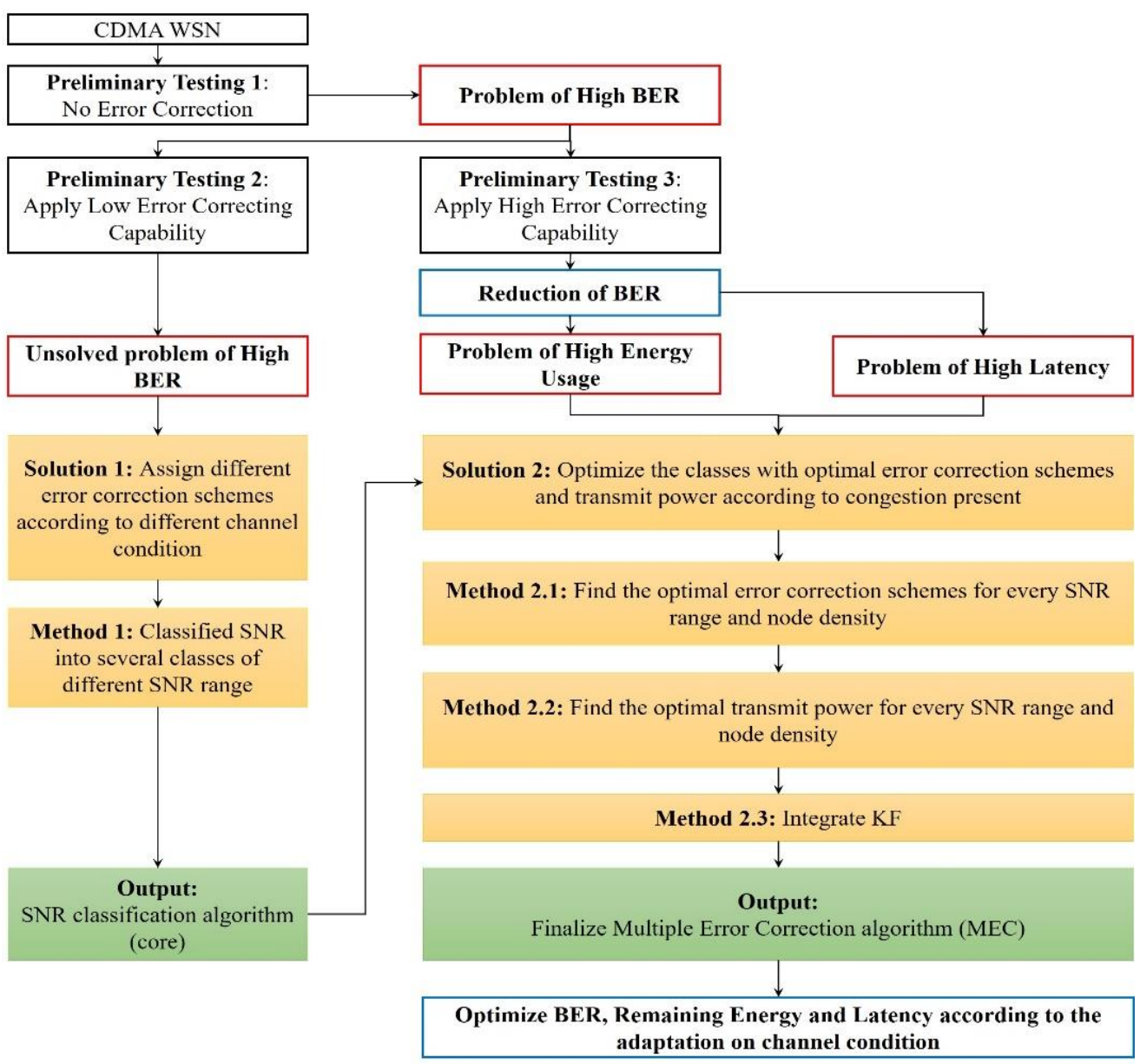

Figure 1. Chronology of the development of Multiple Error Correction (MEC)

From this observation, we concluded that there is the need to use multiple coding schemes for different range of SNR. We also considered some of other related works [16] which demonstrated that the multi-coding is more energy-efficient. Based on this deduction, we proposed the SNR classification algorithm in adapting to the changes of channel condition. This algorithm follows the existing process of HARQ in which we modified the algorithm to be injected with MEC module and transmit power module. We also integrated Kalman Filter (KF) to estimate the SNR values to avoid assigning wrong error correction due to sudden changes in a noisy channel. Some field tests [17]-[19] observed that the signal impairments is timevarying. In a time-varying condition, there is a need to continuously track the changes within the channel as signals frequently attenuated, reflected, and refracted. In addition, the hardware itself might be a contributing factor to the changes in SNR as different nodes gives out different SNR. Thus, channel estimation is substantial to ensure that proper error correcting codes assigned to a defined SNR range which corresponds to the sudden changes in SNR when there are transmissions between nodes in any given time. KF is among the common state estimator with advantages such that low complexity compared to other filters [20] and its capability to provide better estimation for Gaussian and linear models, and limited non-linearity. We integrated KF prior to generating data and after the decoding process. In order to minimize the computational overhead of KF equation, we modified the ACK/NACK message which append the SNR value of the estimation initiated to the transmitted bits from the sender. This is to acknowledge the receiver on the previous estimation value and to prepare the transmission if there are sudden changes in SNR value so that the possibility in assigning wrong error correcting codes is reduced if the sender initiates another transmission.

\subsection{SNR Classification towards the Adaptation to the Changes in Channel Condition}

We divided the methodology into two following solutions based on the unsolved problems of high BER and the problems from high energy usage and high latency due to the implementation of high error 
correcting capability (Figure 1). In method 1, we proposed the channel adaptation algorithm based on HARQ process and classified the range of SNR. We calculated the range of SNR for our predefined topologies of random uniform node distribution and random non-uniform node distribution. A random uniform node distribution consists of layered topology with random node scattering around the sink with uniform distance of $10 \mathrm{~m}$ between each node. Meanwhile, the random non-uniform distribution consists of layered topologies where the distance between nodes varied between 10-100 m. The SNR was calculated following the standardised equation below [21]:

$$
S N R(d B)=P_{\text {signal }}-P_{\text {noise }}
$$

where $P_{\text {signal }}$ denotes the received signal power while $P_{\text {noise }}$ denotes the noise power in which that $P$ is measured in units of power (Watts or milliwatts). Extending the formula, the signal power can be obtained using Friis Transmission equation as shown in Equation (2) [22]:

$$
\mathrm{P}_{\text {signal }}=\frac{\left(\mathrm{P}_{\mathrm{T}} \mathrm{G}_{\mathrm{T}} \mathrm{G}_{\mathrm{R}}\right)\left(\lambda^{2}\right)}{(4 \pi \mathrm{R})^{2}}
$$

where $P_{T}$ is transmit power, $G_{T}$ is the gain of transmit antenna, $G_{R}$ is the gain of receiving antenna, and $R$ is the distance between sender and receiver. The noise power, $P_{\text {noise }}$ is calculated following Equation (3) [23].

$$
\mathrm{N}_{0}=\mathrm{kT}_{\mathrm{s}} \mathrm{B}
$$

where $\mathrm{k}$ denotes the Boltzmann constant, $\mathrm{T}_{\mathrm{s}}$ denotes the system temperature, and B denotes the bandwidth.

From the calculation, we obtained the actual SNR that follows our predefined architecture and parameters as shown in Table 1 (Section 2.3). We extracted and applied the calculated values into the prebuilt system identification model using MATLAB. From the extracted data, we obtained the matrices from the best fit model using the input-output model from the system identification tool; matrices A ($0.236468303496098), \mathrm{B}(0.113429553384058)$, and C (3.59450124244613). Table 1 shows the extracted information of actual SNR alongside with the estimated SNR obtained using Kalman Filter equation as shown in Equation (4) and Equation (5).

$$
\begin{aligned}
& \mathrm{X}_{\mathrm{k}}=\mathrm{Ax}_{\mathrm{k}-1}+\mathrm{Bu}_{\mathrm{k}-1}+\mathrm{w}_{\mathrm{k}-1} \\
& Y_{k}=H x_{k}+v_{k}
\end{aligned}
$$

where $\mathrm{A}$ is the state transition model applied to the previous state $\mathrm{x}_{\mathrm{k}-1}, \mathrm{~B}$ is the control-input model applied to the control vector $\mathrm{u}_{\mathrm{k}-1}$, and $\mathrm{w}$ is the process noise. $H x_{k}$ denotes the observation model which maps the true state space into the observed space and $v_{k}$.

Table 1 shows the actual SNR obtained with the estimated SNR values after we have calculated the measurement and innovative gain from KF equation. We then divided the SNR range into five distinct groups: $\mathrm{SNR}_{1 \mathrm{w}}$ (SNR lowest) with the range of $\mathrm{SNRv} \leq 5, \mathrm{SNR}_{1}\left(\mathrm{SNR}\right.$ low) with $6 \leq \mathrm{SNRv} \leq 20, \mathrm{SNR}_{\mathrm{m}}(\mathrm{SNR}$ medium) with $21 \leq \mathrm{SNRv} \leq 35, \mathrm{SNR}_{\mathrm{h}}$ (SNR High) with $36 \leq \mathrm{SNRv} \leq 50$, and $\mathrm{SNR}_{\mathrm{hg}}$ (SNR highest) where $\mathrm{SNRv} \geq 51$. Based on previous studies and real-time testing [24], most studies agreed that the link is in a good quality when the SNR value is more than $40 \mathrm{~dB}$. Thus, we set the benchmark of SNR in high condition $\left(\mathrm{SNR}_{\mathrm{h}}\right)$ which is considered as good link quality. According to the study [25], SNR value below $5 \mathrm{~dB}$ is considered as bad link quality as the Packet Delivery Ratio (PDR) is zero. According to some studies, SNR alone is not sufficient to indicate congestion when high number of nodes present in one monitoring area. Thus, we implemented the congestion detection based on increasing node density as a network will be congested when node density increases above certain point. A network is assumed to be congested when there are more than 32 nodes in one given area based on a previous study [26]. The PDR and throughputs started to decrease indicating congestion as the node density reaches 45 . Therefore, we noticed that the network started to congest when it reached 48 nodes for our pre-defined topology (300 m x $300 \mathrm{~m}$ ) of the monitoring area. We extended the classes from previous SNR range mentioned where we indicated that the network is extremely congested (EC) when the node density is more than 27.9253, medium congested (MC) when the range of node density is between 16.7552 and 22.3402, and non-congested (NC) when the node density is less than 11.1701 . 
Table 1. The values of actual SNR and estimated SNR with increasing distance between nodes

\begin{tabular}{cccc}
\hline Distance $(\mathrm{m})$ & Transmit Power $(\mathrm{dBm})$ & Actual SNR $(\mathrm{dB})$ & Estimated SNR $(\mathrm{dB})$ \\
\hline 10 & 0 & 41.9764 & 41.9843 \\
20 & 0 & 31.4403 & 31.4482 \\
30 & 0 & 25.2771 & 25.2850 \\
40 & 0 & 20.9043 & 20.9122 \\
50 & 0 & 17.5124 & 17.5203 \\
60 & 0 & 14.7411 & 14.7490 \\
70 & 0 & 12.3979 & 12.4058 \\
80 & 0 & 10.3682 & 10.3761 \\
85 & 0 & 9.4467 & 9.4546 \\
90 & 0 & 8.5779 & 8.5858 \\
100 & 0 & 6.9764 & 6.9843 \\
\hline
\end{tabular}

\subsection{Selection of the Optimal Error Correcting Codes and Transmit Power}

Method 2 was divided into three sub-methods of Method 2.1, Method 2.2, and Method 2.3. Following the classification of SNR range in Method 1, we simulated the error correction codes of BCH and RS with a variety of error correcting capabilities such that $1 \leq \mathrm{t} \leq 10$. We implemented two types of error correcting codes ( $\mathrm{BCH}$ and $\mathrm{RS}$ codes) following the range of codeword length, $n$ denoted as $15 \leq n \leq 127$ for random uniform distribution and $63 \leq n \leq 127$ for random non-uniform node distribution. The idea is to simulate each error correcting code in obtaining the most optimal code with corresponding codeword length and error correcting capability in order to adapt to different channel conditions. For instance, our proposed algorithm implemented high error correcting capability for lower SNR to significantly reduce the too high BER whereas for a good network condition, we implemented a much lower error correcting capability. The reason is that when the SNR condition is good (good BER performance), too high error correcting capability might not be necessary as retransmission is enough to solve the errors. This reduces the overhead of encoding and decoding processes of the ECC as well as the latency caused by the computation of that ECC.

Table 2 and Table 3 show the lists of tested BCH and RS with respective codeword length and error correcting capability. The codes were simulated with CDMA WSN to study the effects of BER and remaining energy as well as latency and to find the most optimal error correcting codes for every range of SNR that had been classified. We also added the transmit power calibration to our proposed algorithm to maximize remaining energy and reduce noises in congested area (Method 2.2). In a condensed network where the nodes are so close with each other and having a good range of transmission coverage, implementing high transmit power might not be necessary.

Table 2. The List of Simulated BCH codes

\begin{tabular}{cccc}
\hline $\begin{array}{c}\text { Error Correcting } \\
\text { Codes }\end{array}$ & $\mathrm{n}$ & $\mathrm{k}$ & $\begin{array}{c}\text { Error Correcting } \\
\text { Capabilities, t }\end{array}$ \\
\hline & 7 & 4 & 1 \\
& 15 & 5 & 3 \\
& 31 & 26 & 1 \\
& 31 & 16 & 3 \\
& 31 & 11 & 5 \\
$\mathrm{BCH}$ & 63 & 57 & 1 \\
& 63 & 45 & 3 \\
& 63 & 24 & 7 \\
& 63 & 18 & 10 \\
& 127 & 120 & 1 \\
& 127 & 92 & 5 \\
& 127 & 64 & 10 \\
& 255 & 247 & 1 \\
& 255 & 179 & 10 \\
& 511 & 502 & 1 \\
& 511 & 466 & 5 \\
& 511 & 421 & 10 \\
\hline
\end{tabular}

Table 3. The List of Simulated RS codes

\begin{tabular}{cccc}
\hline $\begin{array}{c}\text { Error Correcting } \\
\text { Codes }\end{array}$ & $\mathrm{n}$ & $\mathrm{k}$ & $\begin{array}{c}\text { Error Correcting } \\
\text { Capabilities, } \mathrm{t}\end{array}$ \\
\hline & 7 & 3 & 1 \\
& 15 & 11 & 2 \\
& 15 & 5 & 5 \\
& 31 & 27 & 2 \\
& 31 & 13 & 9 \\
& 31 & 11 & 10 \\
$\mathrm{RS}$ & 63 & 59 & 2 \\
& 63 & 45 & 9 \\
& 63 & 43 & 10 \\
& 127 & 123 & 2 \\
& 127 & 113 & 7 \\
& 127 & 107 & 10 \\
& 255 & 251 & 2 \\
& 255 & 235 & 10 \\
& 511 & 507 & 2 \\
& 511 & 499 & 6 \\
& 511 & 491 & 10 \\
\hline
\end{tabular}

In addition, in this condensed network, the nodes might be interfering with the transmission range of another nodes [8],[9]. This promotes the increase in interferences and noises and yet the reduction of transmit power might be able to reduce the Multiple Access Interference (MAI) from the CDMA architecture. In a condensed network where generally the high number of nodes with high transmit power might lead the MAI to peak [27],[28], causing the packet to be corrupted and increase the BER. The reduction in transmit power might be able to slightly increase the remaining energy [29] and theoretically might be able to reduce MAI as 
well. However, we also suggested that the transmit power reduction must be optimal as too much reduction of transmit power will also causes packet drop as the signal power decreases whereas the distance between nodes in the non-random uniform distribution increase to $100 \mathrm{~m}$. The transmit power can be calculated by using the Friis Transmission equation shown in Equation (2). Equation (6) denotes the simplified equation to obtain transmit power from the received signal power:

$$
P t=\operatorname{Pr} x\left(4 \pi d^{2}\right)
$$

where $\operatorname{Pr}$ is the received signal and $d$ is the distance between nodes.

\subsection{Measurement Models}

Table 4 shows the parameters defined for testing and simulation of the proposed work. We implemented the minimum distance of $10 \mathrm{~m}$ for uniform distribution and $100 \mathrm{~m}$ for non-uniform distribution. Non-uniform distribution is referred to as the distance between nodes (might not be the same from one node to another) resulting in different levels of node density present within one monitoring area.

Table 4. Parameters defined for simulation model

\begin{tabular}{|c|c|}
\hline Parameter & Value \\
\hline Min. dist. between two nodes & $10 \mathrm{~m}$ \\
\hline Noise & AWGN \\
\hline Transmit Power $\left(\mathrm{p}_{\mathrm{t}}\right)$ & $\begin{array}{l}\text { Power allocation depending on SNR estimation (-10 } \\
\mathrm{dBm} \text { to } 0 \mathrm{dBm})\end{array}$ \\
\hline Monitoring area $\left(\right.$ meter $\left.^{2}\right)$ & $300 \mathrm{~m} \times 300 \mathrm{~m}$ \\
\hline Path loss parameter $(\alpha)$ & 3.5 \\
\hline Payload, Header & $128,256 \mathrm{bit} / \mathrm{pkt}$ \\
\hline NACK/ACK $\left(n_{b}\right)$ & $\begin{array}{l}8 \text { bits (additional } 6 \text { bits as NACK/ACK were appended } \\
\text { with SNR and update value from the receiver) }\end{array}$ \\
\hline Error Detection & CRC-30 (CDMA compliance) \\
\hline Error Correction & Proposed MEC (variation on the $\mathrm{BCH}$ and RS ECC) \\
\hline Number of Nodes & $4,16,32,48,64,80$ \\
\hline Number of Bits (bits) & $10000,20000,30000,40000,50000$ \\
\hline
\end{tabular}

The application of $100 \mathrm{~m}$ (as the maximum distance between nodes) supports the common sensor nodes such as MicaZ and TelosB with the transmission range up to $100 \mathrm{~m}$. We added Additive White Gaussian Noise (AWGN) and Rayleigh Fading to study the impacts between these two interventions. The power allocation was calibrated according to the SNR range as well as error correction schemes.

We developed our measurement model to measure the performances of CDMA WSN in terms of remaining energy, BER, and latency. The following equations were used to obtain the measurement results. The expression of remaining energy including decoding energy can be denoted as:

$$
\text { Energy } y_{\text {Ecc }}=\text { Hop } \times \mathrm{No}_{\text {packet }} \times\left(\mathrm{No}_{\text {bits }}+\left(\mathrm{No}_{\text {bits }} \times 0.75\right)+\text { Energy }_{\text {Dec }}\right.
$$

where Hop corresponds to number of hops of the predefined layered architecture, No $o_{\text {packet }}$ is the number of packets involved in the transmission, $\mathrm{No}_{\text {bits }}$ is number of generated bits which for research (10000 bits), and Energy $_{\text {Dec }}$ is the decoding energy calculated in as shown in equation (8).

$$
\text { Energy } y_{\text {Dec }}=\left(2 \mathrm{nt}+2 \mathrm{t}^{2}\right)\left(\text { Energy } y_{\text {addition }}+\text { Energy } y_{\text {multiplication }}\right)
$$

where $\mathrm{n}$ denotes the codeword length of the selected error correcting codes and $\mathrm{t}$ is the error correcting capability of that error correcting codes. We tabulated the tested codes in which were used in the calculation of decoding energy in Table 2 and Table 3. We also modelled the BER formulation in BPSK modulation as follows:

$$
\mathrm{BER}=\frac{1}{2}\left(1-\sqrt{\frac{\frac{\mathrm{E}_{\mathrm{b}}}{\mathrm{N}_{0}}}{\frac{\mathrm{E}_{\mathrm{b}}}{\mathrm{N}_{0}}+1}}\right)
$$

Latency is calculated following Equation (10), where Delay Propopagation $_{\text {is }}$ the propagation delay. Propagation delay refers to the time taken between departure of data from the sender and arrival of data at the 


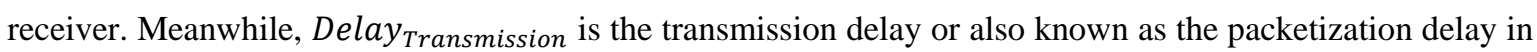
which can be defined as the amount of time required to transmit all of the packet's bits into the link. Usually, the transmission delay is affected by data-rate of the link. Propagation delay was calculated by dividing the distance between sender and receiver with propagation speed of the media. The transmission delay was calculated by dividing the length of packet in bits with the transmission rate of the predefined network.

$$
\text { Latency }=\text { Delay }_{\text {Propagation }}+\text { Delay }_{\text {Transmission }}
$$

\subsection{Optimal Error Correction Schemes for the proposed Multiple Error Correction (MEC)}

Table 5 shows the settings of optimal error correction schemes for the proposed Multiple Error Correction (MEC) algorithm. This setting is optimal if the architecture follows our predefined topologies in which the default number of bits is 10000 bits with increasing number of nodes (between 4 and 80 nodes). In this study, the CDMA architecture implemented Multi-Carrier (MC-CDMA) channel access method and BPSK modulation as tabulated in Table 4. From the simulation results acquired, the BCH codes with codeword length of 127 cannot outperform RS codes with the same codeword length when the network condition is extremely congested due to redundancy added by the $\mathrm{BCH}$ codes is higher than the RS codes. In some studies, more redundancies might lower the BER. However, in our case, too high redundancy appended to the transmitted bits in a congested network flooded the network causing more bits to be corrupted. Thus, we observed that RS code with codeword length of 127 is optimum for an extremely congested condition. For a medium congested condition, we applied BCH codes for both, high SNR and the highest SNR with good link condition where BER is relatively low. Higher appended bits might not corrupt the bits as much as when the SNR is low. For a non-congested condition, we applied retransmission for higher SNR with low BER present in the network as the retransmission is enough to correct the erroneous bits. This is also to add up that there were only a few nodes that might interfere with the transmission range in which if there were collisions or interference that can corrupt the bits, the errors might not be too significant compared to the condition with higher node density.

Table 5. Optimal error correcting codes for MEC

\begin{tabular}{|c|c|c|c|c|}
\hline SNR classes extension & $\begin{array}{c}\text { Error Correcting } \\
\text { Codes }\end{array}$ & $\begin{array}{c}\text { Codeword length, } \\
n\end{array}$ & $\begin{array}{c}\text { Information Bits, } \\
\mathrm{k}\end{array}$ & $\begin{array}{c}\text { Error Correctins } \\
\text { Capability, } \mathrm{t}\end{array}$ \\
\hline \multicolumn{5}{|l|}{ Condition: EC } \\
\hline Case 1: $\mathrm{ECSNR}_{\mathrm{lw}}$ & RS & 127 & 113 & 7 \\
\hline Case 2: $\mathrm{ECSNR}_{1}$ & RS & 127 & 115 & 6 \\
\hline Case 3: $\mathrm{ECSNR}_{\mathrm{m}}$ & RS & 127 & 117 & 6 \\
\hline Case 4: $\mathrm{ECSNR}_{\mathrm{h}}$ & RS & 127 & 119 & 4 \\
\hline Case 5: ECSNR & RS & 127 & 121 & 3 \\
\hline \multicolumn{5}{|l|}{ Condition: MC } \\
\hline Case 1: $\mathrm{MCSNR}_{\mathrm{lw}}$ & RS & 63 & 53 & 5 \\
\hline Case 2: MCSNR $_{1}$ & RS & 63 & 51 & 6 \\
\hline Case 3: $\mathrm{MCSNR}_{\mathrm{m}}$ & RS & 63 & 49 & 7 \\
\hline Case 4: MCSNR $_{\mathrm{h}}$ & $\mathrm{BCH}$ & 63 & 36 & 5 \\
\hline Case 5: MCSNR $_{\mathrm{hg}}$ & $\mathrm{BCH}$ & 63 & 24 & 7 \\
\hline \multicolumn{5}{|l|}{ Condition: NC } \\
\hline Case 1: MCSNR $_{\mathrm{lw}}$ & $\mathrm{BCH}$ & 127 & 64 & 10 \\
\hline Case 2: $\mathrm{NCSNR}_{1}$ & RS & 127 & 121 & 3 \\
\hline Case 3: $\mathrm{NCSNR}_{\mathrm{m}}$ & $\mathrm{BCH}$ & 31 & 11 & 6 \\
\hline Case 4: $\mathrm{NCSNR}_{\mathrm{h}}$ & RS & 31 & 17 & 7 \\
\hline Case 5: $\mathrm{NCSNR}_{\mathrm{hg}}$ & \multicolumn{4}{|c|}{ Retransmission with IR } \\
\hline
\end{tabular}

Table 6 shows the optimal transmit power corresponding to different classes and congestion present based in our abovementioned architecture. The transmit power with high SNR were significantly reduced to $20 \%$ for extremely congested condition, $40 \%$ for medium congested, and $50 \%$ for non-congested. In the extremely congested condition, the transmit power was not reduced too much as compared to the noncongested condition in order to avoid increment in the noise present. This is because the signal power will reduce the transmit power calibrated. The lower power will be peak the noise, which lead to the increment in BER. For non-congested condition, the transmit power can be reduced until 50\% as having high SNR indicate high signal power with low noise power. 
Table 6. Optimal transmit power for MEC

\begin{tabular}{|c|c|}
\hline SNR classes extension & Transmit power calibration \\
\hline \multicolumn{2}{|l|}{ Condition: EC } \\
\hline Case 1: $\mathrm{ECSNR}_{\mathrm{lw}}$ & Maintain \\
\hline Case 2: ECSNR & Maintain \\
\hline Case 3: $\mathrm{ECSNR}_{\mathrm{m}}$ & Reduction by $10 \%$ \\
\hline Case 4: $\mathrm{ECSNR}_{\mathrm{h}}$ & Reduction by $10 \%$ \\
\hline Case 5: ECSNR $_{\mathrm{hg}}$ & Reduction by $20 \%$ \\
\hline \multicolumn{2}{|l|}{ Condition: MC } \\
\hline Case 1: $\mathrm{MCSNR}_{\mathrm{lw}}$ & Maintain \\
\hline Case 2: MCSNR $_{1}$ & Reduction by $10 \%$ \\
\hline Case 3: $\mathrm{MCSNR}_{\mathrm{m}}$ & Reduction by $20 \%$ \\
\hline Case 4: MCSNR $_{\mathrm{h}}$ & Reduction by $30 \%$ \\
\hline Case 5: MCSNR $_{\mathrm{hg}}$ & Reduction by $40 \%$ \\
\hline \multicolumn{2}{|l|}{ Condition: NC } \\
\hline Case 1: $\mathrm{NCSNR}_{\mathrm{lw}}$ & Reduction by $10 \%$ \\
\hline Case 2: NCSNR $_{1}$ & Reduction by $20 \%$ \\
\hline Case 3: $\mathrm{NCSNR}_{\mathrm{m}}$ & Reduction by $30 \%$ \\
\hline Case 4: $\operatorname{NCSNR}_{h}$ & Reduction by $40 \%$ \\
\hline Case 5: NCSNR $_{\mathrm{hg}}$ & Reduction by $50 \%$ \\
\hline
\end{tabular}

\section{RESULTS AND ANALYSIS}

We collected the results based on the experiment of MEC in terms of increment in node density and compared the non-channel adaptation using the existing error correction schemes. Figure 2 shows the average remaining energy against SNR between $\mathrm{MEC}$ with $\mathrm{BCH}(127, \mathrm{k}) \mathrm{t}=10, \mathrm{RS}(127,121) \mathrm{t}=3, \mathrm{BCH}(31, \mathrm{k}) \mathrm{t}=$ 7 , and $\operatorname{RS}(31, \mathrm{k}) \mathrm{t}=7$. The existing $\mathrm{RS}(31, \mathrm{k}) \mathrm{t}=7$ have the highest constant remaining energy throughout the SNR. The highest remaining energy for MEC is obtained when the SNR is at the good condition. Even though RS $(31, \mathrm{k}) \mathrm{t}=7$ had the highest remaining energy in a non-congested condition, the higher redundancy added by this code was not practical for higher node density.

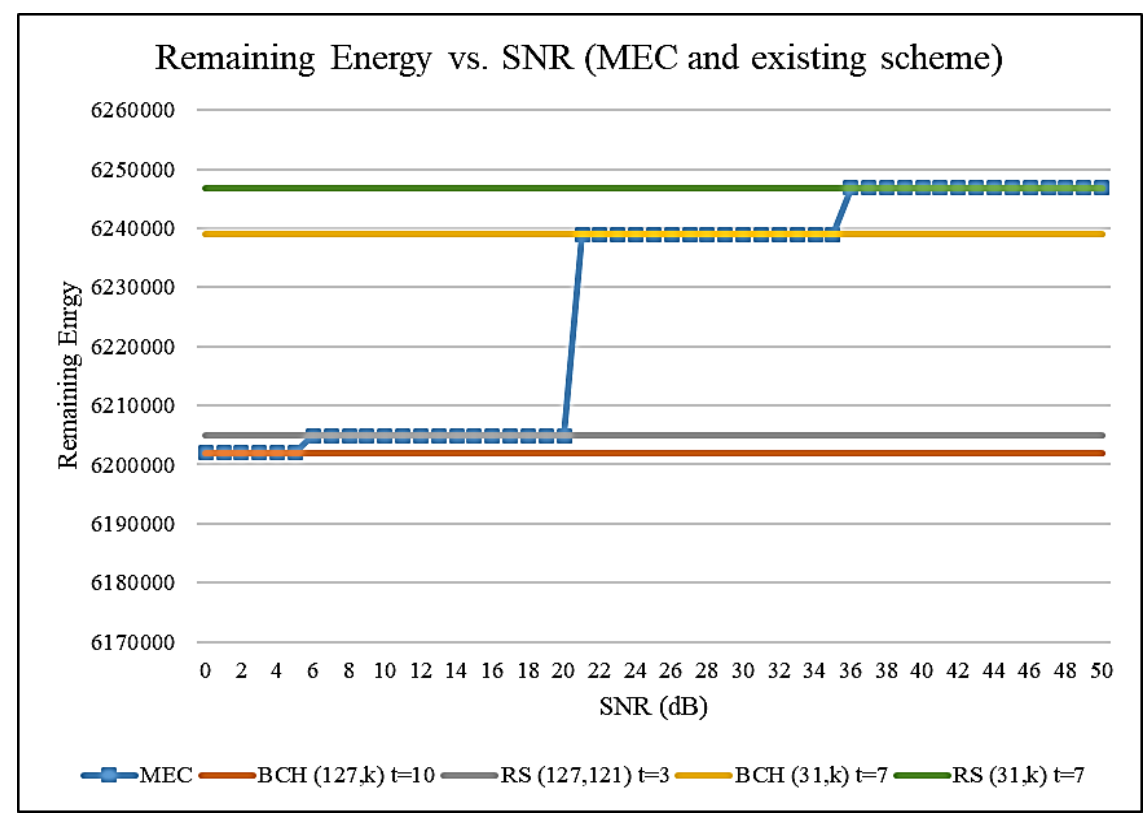

Figure 2. Average Remaining Energy against SNR

In addition, MEC cannot reduce BER as further reduction of the remaining energy was too much due to drawbacks between the capabilities of error corrections that added redundancies with more complexities and required higher energy when better error correction is used. However, MEC optimized the remaining energy in high SNR as too powerful error correction schemes were not necessary. Instead, the retransmission was enough to handle the errors which do not used too much energy as compared to ECC due to decoding and computation overhead. However, for low SNR (where BER is relatively very high), it is substantial to correct the errors to reduce too many resend packets from the sender. A higher number of 
flooded retransmissions in the network not only uses energy but also resources as well which might render the network causing malfunction. The remaining energy for low SNR was lesser compared to other SNR range as higher error correction capability was used. Thus, we can conclude that the optimisation between remaining energy and BER by means of adaptation to the channel condition itself. In low SNR, the remaining energy might not be able to be boosted as much as when the SNR is high. This low error correcting capability codes might not able to handle the high error rates if lower error correcting capability is used in low SNR where BER is high.

Figure 3 shows the comparison of average latency against SNR between MEC and existing codes. The existing coding schemes have constant latency throughout the SNR due to the absence of channel adaptation. The latency changed with SNR range due to the changes in error correction used throughout the SNR as shown in the figure. The latency corresponded to the appended bits in the network where higher appended bits or redundancy causes higher latency as large number of bits prolonged the decoding time of the received information. In this study, $\mathrm{BCH}$ recorded higher latency due to its complexity and higher number of redundancies. However, this corresponds to the fact that $\mathrm{BCH}$ have better error correction capability that the RS code. For instance, $\mathrm{BCH}(31, \mathrm{k})$ outperformed $\mathrm{RS}(31, \mathrm{k})$ with the same $\mathrm{t}=7$ in terms of BER even though the latency was too high. However, the use of multi-coding might able to optimize the latency as the latency will not always be high throughout the SNR. When high redundancy is not needed, the latency will be reduced by using much lower capability codes that appended much lower redundancy.

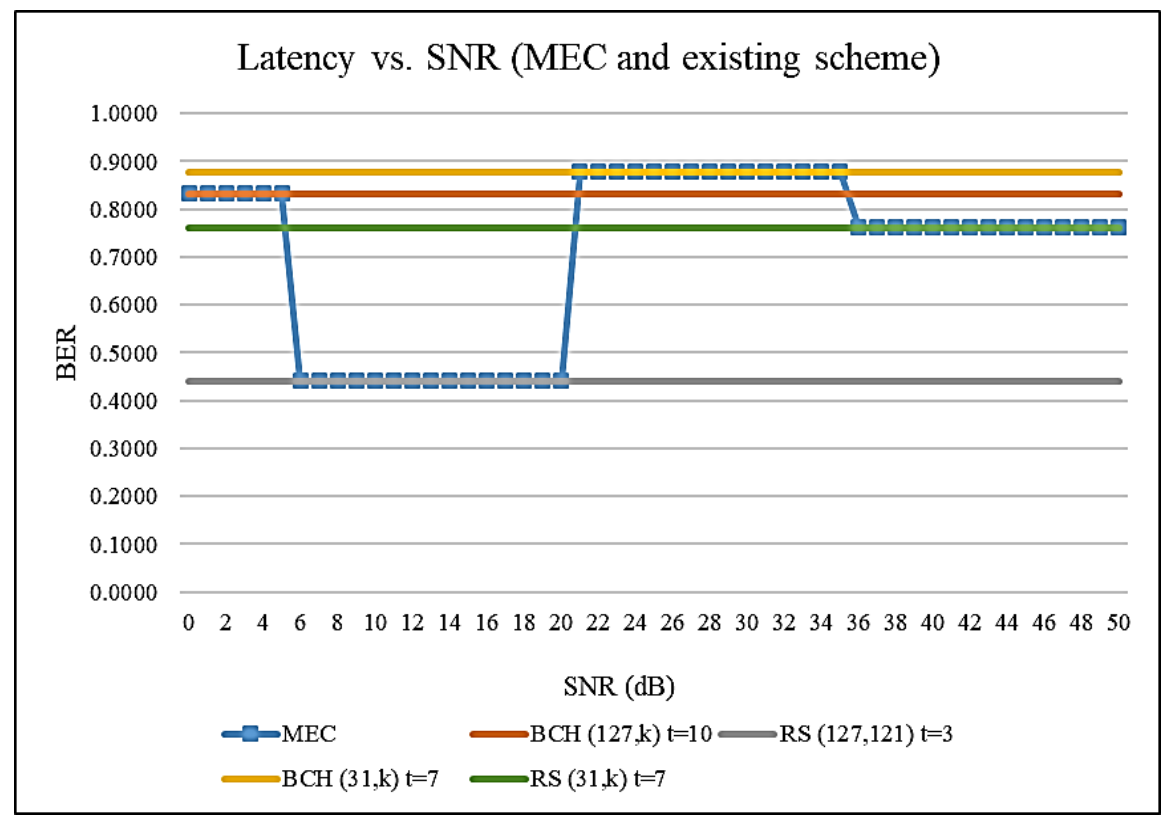

Figure 3. Average Latency against SNR

Figure 4 illustrated the average BER against increasing node density. The MEC showed the lowest BER compared to existing schemes for the non-congested condition of less than 11.1701 node density when SNR reaching $30 \mathrm{~dB}$. The reason is that $\mathrm{BCH}(31, \mathrm{k})$ was implemented for medium SNR in non-congested environment as such redundancy significantly solve the BER and does not flood the network in the noncongested environment. However, for higher node density, $\mathrm{BCH}(31, \mathrm{k})$ was impractical because of too high redundancy. However, the existing RS $(127, \mathrm{k})$ did not outperform BER of MEC for non-congested condition $(\leq 11.1701)$.

Figure 5 shows the average remaining energy against node density. The network achieved the highest remaining energy in congested condition for medium SNR as we used medium error correcting capability which optimised the need between remaining energy and redundancy added to the network. There was much higher error correcting capability used for low congested condition as the remaining energy was slightly lower than the existing RS $(31, \mathrm{k})$. However, in comparison to Figure 4 where BER of MEC was at its lowest when node density at 11.1701, the higher remaining energy of RS proved that the existing RS did not optimize the BER and remaining energy. Meanwhile, there was slight increment in the remaining energy from $628946.33 \mathrm{~J}$ for node density between 16.7552 and 22.3402 . 


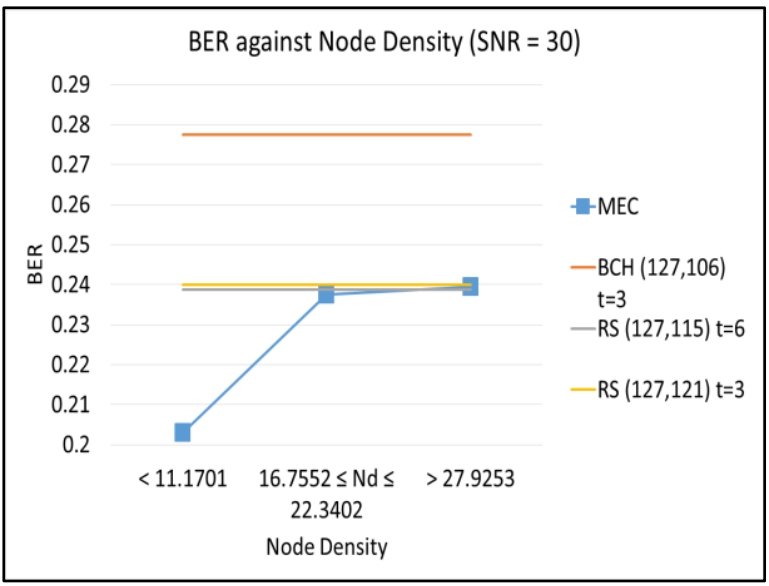

Figure 4. Average BER against Node density (for $\mathrm{SNR}=30$ )

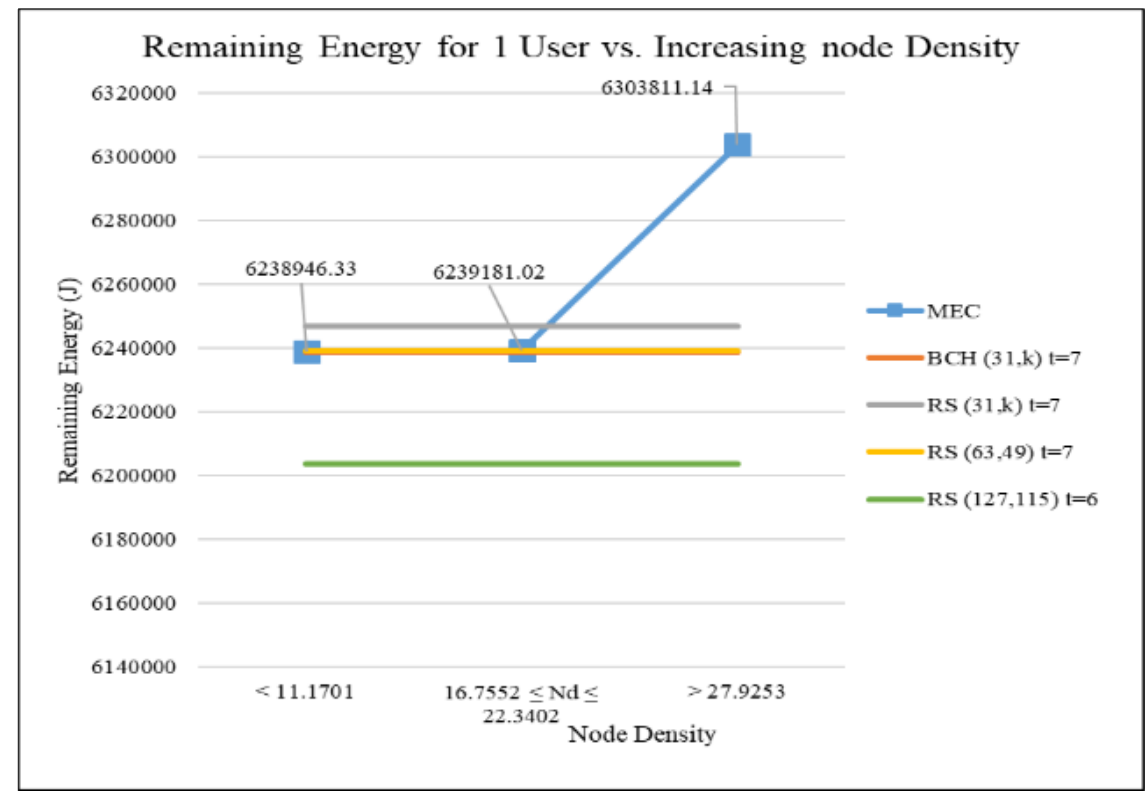

Figure 5. Average Remaining Energy against Node density

Figure 6 shows the Percentage increment in remaining energy of MEC against SNR compared with $\mathrm{BCH}(127, \mathrm{k})$ for $\mathrm{t}=7$, RS $(127, \mathrm{k})$ for the same $\mathrm{t}$ and the comparison of increment in remaining energy between existing RS and $\mathrm{BCH}$. From the graph, it can be observed that as the SNR increased, the percentage increment in remaining energy also increased. Besides that, the percentage of increment of remaining energy of MEC is higher than the existing RS $(127, \mathrm{k})$ and BCH $(127, \mathrm{k})$ when SNR achieving between $20 \mathrm{~dB}$ and $50 \mathrm{~dB}$.

It is also pointed out that the RS $(127, \mathrm{k})$ has a higher percentage of increment during the low SNR of $20 \mathrm{~dB}$ because MEC has more powerful error correction schemes which used more energy. However, the difference between the percentage increment of MEC and RS is quite small. This also corresponds to the graph in Figure 4 which demonstrated that during the lowest SNR, MEC achieved better BER than the existing RS which results in lower remaining energy. Thus, a conclusion was made where MEC had optimised the performances between the BER and remaining energy and for the lowest SNR and for highest SNR, MEC performed more towards optimisation of remaining energy and latency. 


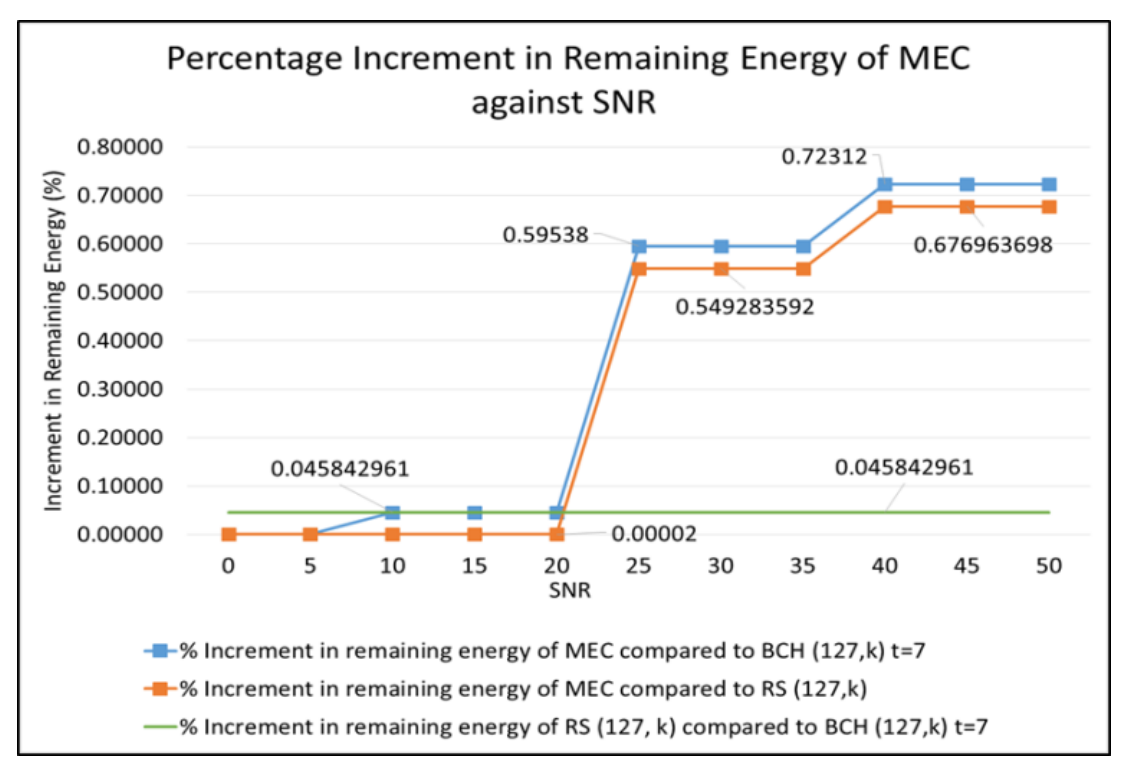

Figure 6. Percentage increment in remaining energy of MEC against SNR

\section{CONCLUSION}

This paper developed the algorithm towards adapting the changes in channel condition and congestion by classifying SNR into several ranges in order to optimise the performances between remaining energy and BER. Our proposed algorithm merits on its capability to adapt to the changes in SNR and optimise the performance of remaining energy and BER in low SNR as well as high SNR in which every SNR range describe different error correction used and transmit power calibration to minimise the higher redundancies when these excessive redundancies are not needed in better SNR condition. Transmit power calibration aided to reduce the interferences and noises in noisy channel when SNR is low. For example, when SNR was at its highest, we assumed the network condition was good and retransmission was likely more sufficient to correct the corrupted bits compared to error correcting codes. While, when SNR is low, the noise ratio usually fluctuated and causes the BER to rises which in need for higher error correcting capability. Relating to our analysis, we had obtained lower BER during low SNR compared to the existing method in which the error correction was constant throughout the increasing SNR. While, we are able to maintain remaining energy and BER by using medium error correcting capability during the medium and high SNR condition as for existing method, higher error correcting capability such as $\mathrm{BCH}(127, \mathrm{k})$ might not be necessary as demonstrated have the lowest remaining energy in Figure 2 even when the SNR is high. In addition, MEC outperformed the existing $\mathrm{BCH}$ as the number of nodes increased because MEC implemented RS $(63,57)$ during medium SNR corresponding to the fact that the RS codes in MEC possessed lower latency than $\mathrm{BCH}$ due to its lower error correction capability that appended much lower number of redundancies than $\mathrm{BCH}$. However, in a medium network condition of SNR, it was observed that RS corrected the errors as well as $\mathrm{BCH}$ since the BER was optimised and significantly reduced.

As for the future work, it is suggested to study the multi-coding schemes of MEC with other error correcting codes such as Turbo codes, Hamming, and Low-Density Parity Check (LDPC) codes. Last but not least, it is recommended that future research to include the aspects of localisation of nodes as well as mobility since the study of localisation promotes better scalability with different geographical limitations assessment. Besides, mobility might further improve MEC in supporting different types of environmental monitoring other than natural disasters such as pollution monitoring, agricultural monitoring, and even human sensing that requires node mobility features.

\section{ACKNOWLEDGEMENTS}

This research is supported by the Research Management Institute, Universiti Teknologi Mara and Ministry of Higher Education (MOHE) registered under the Fundamental Research Grant Scheme (FRGS) \#600-RMI/FRGS 5/3 (46/2015). 


\section{REFERENCES}

[1] C. Hung and C. Hsieh, "Big Data Management on Wireless Sensor Networks," Big Data Analytics for SensorNetwork Collected Intelligence, pp. 99-116, 2017.

[2] S. Rani, et al., "Can sensors collect big data? an energy-efficient big data gathering algorithm for a WSN," IEEE Transactions on Industrial Informatics, vol/issue: 13(4), pp. 1961-1968, 2017.

[3] M. Kocakulak and I. Butun, "An overview of Wireless Sensor Networks towards internet of things," 2017 IEEE 7th Annual Computing and Communication Workshop and Conference (CCWC), pp. 1-6, 2017.

[4] N. Khalil, et al., "Wireless sensors networks for Internet of Things," 2014 IEEE Ninth International Conference on Intelligent Sensors, Sensor Networks and Information Processing (ISSNIP), pp. 1-6, 2014.

[5] G. Song, et al, "A Mobile Sensor Network System for Monitoring of Unfriendly Environments," Sensors. Molecular Diversity Preservation International (MDPI), vol/issue: 8(11), pp. 7259-7274, 2018.

[6] M. M. Y. Masood, et al., "A Kalman filter based adaptive on demand transmission power control (AODTPC) algorithm for wireless sensor networks," 2012 International Conference on Emerging Technologies. IEEE, pp. 1-6, 2012.

[7] V. Venugopal and S. Ramakrishnan, "Received signal strength index estimation using Kalman Filter for fuzzy based transmission power control in wireless sensor networks," 2014 International Conference on Control, Instrumentation, Communication and Computational Technologies (ICCICCT). IEEE, pp. 81-86, 2014.

[8] U. Datta, et al., "Energy level performance of HARQ-II scheme in CDMA wireless sensor network with correlated interferers," Proceedings - 2011 Annual IEEE India Conference: Engineering Sustainable Solutions, INDICON2011, vol. 2, pp. 1-4, 2011.

[9] U. Datta and S. Kundu, "Performance of multi-hop CDMA wireless sensor networks with correlated interferers using different retransmission strategies and error control schemes," International Journal of Sensor Networks, vol. 15, pp. 40, 2014.

[10] N. Ali, et al, "Single and Multi Coding Schemes for Efficient Wireless Sensor Networks," Wireless Sensor Network, vol. 4, pp. 52-58, 2012.

[11] S. Razali, et al., "Performance of Multiple Error Correction (MEC) Scheme Based Hybrid ARQ (HARQ) Algorithm for Maximizing Lifetime of Wireless Sensor Network (WSN) for Natural Disaster Monitoring," Journal of Fundamental and Applied Sciences, vol/issue: 9(5S), pp. 336-351, 2017.

[12] S. M. Razali, et al., "Implementation of Hybrid ARQ (HARQ) error control algorithm for lifetime maximization and low overhead CDMA Wireless Sensor Network (WSN)," 2016 IEEE Conference on Wireless Sensors (ICWiSE). IEEE, pp. 71-76, 2016.

[13] S. Razali, et al., "Optimization of Remaining Energy and Error Rates for Wireless Sensor Network," Alfred, R. et al., "Computational Science and Technology," Singapore: Springer Singapore, pp. 130-140, 2018.

[14] M. R. Islam, "Error Correction Codes in Wireless Sensor Network: An Energy Aware approach," International Journal of Electrical, Computer, Energetic, Electronic and Communication Engineering, vol. 4, pp. 26-31, 2010.

[15] F. R. Lone, et al., "Performance Comparison of Reed Solomon Code and BCH Code over Rayleigh Fading Channel," International Journal of Computer Applications, vol. 71, pp. 23-26, 2013.

[16] N. Ali, "Single and Multi Coding Schemes for Efficient Wireless Sensor Networks," Wireless Sensor Network, vol. 04, pp. 52-58, 2012.

[17] D. Sexton, et al, "Radio Channel Quality in Industrial Wireless Sensor Networks," 2005 Sensors for Industry Conference, pp. 88-94, 2005.

[18] L. Tang, et al, "Channel Characterization and Link Quality Radio for Factory Environments," IEEE Transactions on Industrial Informatics, vol/issue: 3(2), pp. 99-110, 2007.

[19] F. Qin, et al., "Effective-SNR estimation for wireless sensor network using Kalman filter," Ad Hoc Networks, vol/issue: 11(3), pp. 944-958, 2013.

[20] A. Doucet and A. M. Johansen, "A Tutorial on Particle filtering and smoothing: Fiteen years later," The Oxford handbook of nonlinear filtering.

[21] M. Finley, "SNR Calculation: Project Manual."

[22] P. Bevelacqua, "The Friis Equation." Available at: http://www.antenna-theory.com/basics/friis.php (Accessed: 6 September 2018).

[23] T. M. Amjad, et al., "Positioning of a Wireless Relay Node for Useful Cooperative Communication," Indonesian Journal of Electrical Engineering and Computer Science, vol/issue: 10(3), pp. 959-965, 2018.

[24] J. Geier, "How to: Define Minimum SNR Values for Signal Coverage." Available at: http://www.wirelessnets.com/resources/tutorials/define_SNR_values.html (Accessed: 5 May 2017).

[25] W. L. Tan, et al., "SNR-Based Link Quality Estimation," 2012 IEEE 75th Vehicular Technology Conference (VTC Spring), pp. 1-5, 2012.

[26] M. M. Chitlange and V. S. Deshpande, "Effect of node density on congestion in WSN," 2015 International Conference on Pervasive Computing (ICPC), pp. 1-3, 2015.

[27] H. S. Gill, et al., "Reduction of Multiple Access Interference in CDMA by using Improved Minimum Mean Square Error Receiver," International Journal of Scientific \& Engineering, vol. 2, pp. 2-5, 2011.

[28] S. J. Honade and D. P. V. Ingole, "Removal of Multiple Access Interference in DS-CDMA System," International Journal of Scientific and Research Publications, vol/issue: 2(6), pp. 1-6, 2012.

[29] H. Cotuk, et al, "The Impact of Transmission Power Control Strategies on Lifetime of Wireless Sensor Networks," IEEE Transactions on Computers, vol/issue: 63(11), pp. 2866-2879, 2014. 


\section{BIOGRAPHIES OF AUTHORS}

\begin{tabular}{|l|l|}
\hline & $\begin{array}{l}\text { Samirah Nasuha Binti Mohd Razali obtained Bachelor of Science in Data Communication and } \\
\text { Networking (Hons.) (2013) from Universiti Teknologi MARA, Perlis, MSc in Computer } \\
\text { Networking (2015) from Universiti Teknologi MARA, Shah Alam and currently doing her } \\
\text { PhD in Computer Science (2015 2018) at the Universiti Teknologi MARA, Shah Alam. Her } \\
\text { PhD research involves several methods in Error Correction Schemes and SNR Estimation } \\
\text { includes SNR classification, multi-coding schemes and adaptive transmit power. }\end{array}$ \\
$\begin{array}{l}\text { Kamaruddin Mamat (Dr.) is a senior lecturer at the Department of Computer and } \\
\text { Mathematical Sciences in Universiti Teknologi of MARA, Shah Alam. He obtained his PhD } \\
\text { in Multimedia Education, Universiti Pendidikan Sultan Idris, Tanjung Malim in 2007. His } \\
\text { research interest involving network management, multimedia network, wireless network and } \\
\text { others. }\end{array}$ \\
$\begin{array}{l}\text { Nor Shahniza Kamal Bashah (Dr.) is a senior lecturer at the Department of Computer and } \\
\text { Mathematical Sciences and currently attached as Head of Strategic Planning. She obtained her } \\
\text { PhD in Telematics from Norwegian University of Science and Technology (NTNU), Norway } \\
\text { on February 2013. Her PhD research involved aorund Service discovery in mobile } \\
\text { environment and her research interest involved in the field of Mobile and Wireless } \\
\text { Communication and Semantic Web. }\end{array}$ \\
\hline
\end{tabular}

